\title{
Certolizumab for the treatment of psoriasis and psoriatic arthritis: a real-world multicentre Italian study
}

\author{
A. Dattola, ${ }^{1,}{ }^{*}$ iD A. Balato, ${ }^{2}$ iD M. Megna, ${ }^{3}$ iD P. Gisondi, ${ }^{4}$ (iD G. Girolomoni, ${ }^{4}$ (iD C. De Simone, ${ }^{5,6}$ \\ G. Caldarola, ${ }^{5}$ iD E. Cama, ${ }^{7}$ S. Piaserico, ${ }^{7}$ M.C. Fargnoli, ${ }^{8}$ iD R. Fidanza, ${ }^{9}$ A. Parodi, ${ }^{10}$ M. Burlando, ${ }^{10}$ \\ A. Offidani, ${ }^{11}$ F. Diotallevi, ${ }^{11}$ C. Potenza, ${ }^{12}$ A. Conti, ${ }^{13}$ A. Chiricozzi, ${ }^{5,6,14}$ E. Campione, ${ }^{1}$ L. Bianchi ${ }^{1}$ \\ ${ }^{1}$ Department of Dermatology, University of Rome, "Tor Vergata", Rome, Italy \\ 2Department of Advanced Biomedical Sciences, University of Naples Federico II, Naples, Italy \\ ${ }^{3}$ Department of Clinical Medicine and Surgery, University of Naples Federico II, Naples, Italy \\ ${ }^{4}$ Section of Dermatology and Venereology, Department of Medicine, University of Verona, Verona, Italy \\ ${ }^{5}$ Institute of Dermatology, Catholic University, Rome, Italy \\ ${ }^{6}$ Dermatology Unit, Fondazione Policlinico Universitario A. Gemelli IRCCS, Rome, Italy \\ ${ }^{7}$ Dermatology Unit, Department of Medicine, University of Padua, Padua, Italy \\ ${ }^{8}$ Department Dermatology, Department of Biotechnological and Applied Clinical Sciences, University of L'Aquila, L'Aquila, Italy \\ ${ }^{9}$ Department Dermatology, San Salvatore Hospital, University of L'Aquila, L'Aquila, Italy \\ ${ }^{10}$ Section of Dermatology, DISSAL, San Martino-IST Polyclinic Hospital,University of Genoa, Genoa, Italy \\ ${ }^{11}$ Dermatology Unit, Department of Clinical and Molecular Sciences, Polytechnic Marche University, Ancona, Italy \\ ${ }^{12}$ Department of Medical-Surgical Sciences and Bio-Technologies, Sapienza University of Rome, Polo Pontino, Terracina, Italy \\ ${ }^{13}$ Dermatology Unit, Surgical, Medical and Dental Department of Morphological Sciences related to Transplant, Oncology and \\ Regenerative Medicine, University of Modena and Reggio Emilia, Modena, Italy \\ ${ }^{14}$ Dermatology Unit, Department of Clinical and Experimental Medicine, University of Pisa, Pisa, Italy \\ *Correspondence: A. Dattola. E-mail: nancydattola@gmail.com
}

\section{Abstract}

Background Certolizumab, a pegylated tumour necrosis factor- $\alpha$ inhibitor, reduced disease activity in randomized trials of patients with psoriasis and psoriatic arthritis. Real-life data are missing.

Objective To confirm the effectiveness and safety of certolizumab in patients with psoriasis and psoriatic arthritis in routine clinical practice.

Methods In this retrospective study involving 11 Italian sites, patients with psoriasis and psoriatic arthritis received subcutaneous certolizumab (400 mg loading dose at 0, 2 and 4 weeks, followed by $200 \mathrm{mg}$ every 2 weeks) for up to 52 weeks. Primary outcomes included mean change from baseline in Psoriasis Area and Severity Index (PASI) and modified Nail Psoriasis Severity Index (mNAPSI) scores, and the proportion of patients achieving a $75 \%, 90 \%$ or $100 \%$ reduction in PASI score. Other endpoints included Disease Activity Score computed on 44 joints correlated with the erythrocyte sedimentation rate during the first hour (DAS44-ESR), Tender Joint Count (TJC), Swollen Joint Count (SJC), pain [visual analogue scale (VAS) score], inflammatory markers and quality of life (QOL).

Results In the study were enrolled 153 patients (mean age: 55 years). Certolizumab reduced the mean PASI score from baseline by $4.45,6.30$ and 7.58 at weeks 12,24 and 52, respectively $(P<0.001$ for all). At weeks 24 and $52,69.6 \%$ and $83.3 \%$ of patients had a PASI score $\leq 3$. DAS44-ESR, TJC, SJC and mNAPSI scores, and pain VAS were also all significantly improved from baseline at each time point. C-reactive protein levels decreased during treatment, being significant at week 24. On multivariate analysis, psoriasis duration, baseline PASI, mNAPSI and pain VAS scores were found to be predictive of the improvement in PASI score at week 12.

Conclusion Certolizumab displayed also in the real-life encouraging results in both psoriasis and psoriatic arthritis patients.

Received: 26 February 2020; Accepted: 21 April 2020

\section{Conflicts of interest}

AD has been a consultant and/or speaker for UCB, Eli Lilly and Leo Pharma; AB has been a scientific consultant/ speaker/clinical study investigator for AbbVie, Celgene, Eli Lilly, Novartis, Pfizer, Sanofi; PG has been a consultant and/ or speaker for AbbVie, Almirall, Celgene, Janssen, Leo Pharma, Eli Lilly, Merck Sharp \& Dohme, Novartis, Pfizer, Sandoz and UCB; GG has been principal investigator in clinical trials sponsored by and/or has received personal fees from AbbVie, Abiogen, Almirall, Amgen, Bayer, Biogen, Bristol-Meyers Squibb, Celgene, Eli Lilly, Galderma, Genzyme, Leo 
Pharma, Menlo therapeutics, Merck, MSD, Novartis, Pfizer, Pierre Fabre, Regeneron, Samsung, Sandoz, Sanofi and Sun Pharma; CDS has been a consultant and/or speaker for AbbVie, Almirall, Biogen, Celgene, Janssen, Leo Pharma, Eli Lilly, Novartis, Pfizer, Sanofi and UCB; SP has been a consultant and/or speaker for AbbVie, Almirall, Celgene, Janssen, Leo Pharma, Eli Lilly, Merck Sharp \& Dohme, Novartis, Pfizer, Sandoz and UCB; MCF has served on advisory boards, received honoraria for lectures and research grants from Almirall, AbbVie, Galderma, Leo Pharma, Mylan, Medac Pharma, Celgene, Pierre Fabre, UCB, Lilly, Pfizer, Janssen, Novartis, Sanofi, Roche and Sun Pharma; RF has served on advisory boards for AbbVie and Novartis; MB has been a consultant and/or speaker for UCB Pharma, Eli Lilly, Leo Pharma, Janssen and Novartis; $A O$ has been a principal investigator for clinical trials sponsored by and/or has received personal fees from AbbVie, Alfasigma, Almirall, Amgen, Difa Cooper, Celgene, Eli Lilly, Galderma, Janssen, Laboratori Farmacologici Milanesi, Leo Pharma, Novartis, Pfizer, Pierre Fabre, Regeneron, Sanofi and UCB; CP has served as speaker and as an advisory board member, received honoraria for lectures and has participated in clinical trials sponsored by Almirall, AbbVie, Alfasigma, Leo Pharma, Celgene, Pierre Fabre, UCB, Eli Lilly, Pfizer, Janssen, Novartis, Sanofi, MSD and Sandoz; A. Conti has been a consultant and/or speaker for AbbVie, Almirall, Amgen, Biogen, Celgene, Janssen, Cilag, Leo Pharma, Eli Lilly, Novartis, MSD, Pfizer, Sandoz and UCB; A. Chiricozzi has served as an advisory board member, has received speaker honoraria or has participated in clinical trials for AbbVie, Almirall, Fresenius Kabi, Janssen, Leo Pharma, Lilly, Novartis, Sanofi Genzyme and UCB; E. Campione has served on advisory boards, received honoraria for lectures and research grants from Celgene, Almirall, Medac Pharma, Alfasigma and Laboratori Farmacologici Milanesi; LB has been a consultant and/or speaker for AbbVie, Celgene, Janssen, Eli Lilly, Novartis, Pfizer, Sanofi and Almirall; AP, FD, MM, GC and E. Cama have no conflicts of interest to declare.

\section{Funding sources}

This study was funded by an unrestricted grant from UCB, who had no role in the design or conduct of the study or preparation of the manuscript, and had no access to study data.

\section{Introduction}

Psoriasis is a chronic inflammatory systemic disease that predominantly affects skin and joints. ${ }^{1-3}$ Evidence for the pathogenesis of psoriasis supports a key role of various inflammatory cytokines, including tumour necrosis factor- $\alpha$ (TNF), interferon- $\gamma$ and interleukin (IL)-17., ${ }^{4,5}$ There are increasing number of effective treatment options for psoriatic patients. ${ }^{2}$ While topical treatments are usually adequate for the control of mild-to-moderate psoriasis, they are often insufficient for moderate-to-severe disease. ${ }^{6}$ Nonbiologic systemic drugs, including methotrexate, cyclosporine, acitretin and apremilast, are established treatments for patients with moderate-to-severe psoriasis and psoriatic arthritis. ${ }^{1,2}$ Additionally, several biologic agents have been developed for the treatment of psoriasis and psoriatic arthritis, including TNF inhibitors (etanercept, infliximab, adalimumab, golimumab and certolizumab), the IL-12/23 inhibitor ustekinumab, the IL-23 inhibitors (guselkumab, tildrakizumab and risankizumab) and IL-17 inhibitors (secukinumab, ixekizumab, which block IL-17A, and brodalumab, which blocks the IL-17 receptor). ${ }^{1,2,7,8}$ Seven of these are currently approved in Europe for the treatment of psoriasis or psoriatic arthritis: adalimumab, etanercept, golimumab, infliximab, ixekizumab, secukinumab and ustekinumab. European guidelines on the management of psoriatic arthritis with pharmacological therapies recommend TNF inhibitors as the first-line biologic in patients eligible for therapy with a biologic agent. ${ }^{9}$ Remarkably, in psoriatic patients loosing response during an anti- psoriatic therapy is a frequent scenario, as well as in psoriatic arthritis. Certolizumab pegol, a pegylated antigen-binding fragment (Fab') of a humanized anti-TNF antibody, is a promising treatment for psoriasis, showing a rapid reduction in disease activity and evidence of long-term clinical efficacy. ${ }^{6,10}$ Certolizumab pegol has a peculiar molecular structure compared to other biologics; it lacks the fragment crystallizable (Fc) region, which limits Fc-mediated cytotoxic effects, ${ }^{11}$ and may also prevent active transplacental transfer to the fetus during pregnancy. ${ }^{11,12}$ In randomized controlled trials, certolizumab treatment resulted in significant and clinically meaningful improvements in symptom severity in patients with psoriatic arthritis ${ }^{13}$ or moderate-to-severe chronic plaque psoriasis. ${ }^{14-16}$ These clinical trial results are supported by preliminary data from real-world studies, in which certolizumab was associated with rapid and significant improvements in joint and skin involvement, as well as improvements in nail psoriasis, and was well tolerated. ${ }^{17-19}$ The aim of our study was to confirm the effectiveness and safety of certolizumab in patients with psoriatic arthritis and psoriasis receiving treatment in a routine clinical practice setting in Italy.

\section{Materials and methods}

\section{Study population}

This retrospective, observational, multicentre study enrolled patients with psoriatic arthritis and psoriasis from 11 institutes 
across Italy. Data regarding patient baseline characteristics, including age, sex, disease characteristics, onset of the disease, comorbidities, previous systemic treatments and any concurrent medications, were recorded.

According to the European and Italian guidelines, ${ }^{20,21}$ patients included in the study had to have been unresponsive or contraindicated to at least two systemic conventional therapies for psoriatic arthritis. Patients with infectious disease, active or latent tuberculosis, human immunodeficiency virus infection, neoplastic disease in the past 5 years, severe heart failure or demyelinating disorders were excluded.

The study was approved by the ethics committee of the coordinating centre (Hospital Tor Vergata, Rome; Approval no. Registro Sperimentazioni 182/18) and was conducted in accordance with the principles of the Declaration of Helsinki and local and international laws.

\section{Study treatment}

Patients received a loading dose of certolizumab $400 \mathrm{mg}$ administered by subcutaneous injection at 0,2 and 4 weeks, after which they received subcutaneous certolizumab $200 \mathrm{mg}$ every 2 weeks for up to 52 weeks.

\section{Clinical assessments}

Clinical evaluations were performed at baseline and weeks 12, 24 and 52. Assessments included medical history, a general and dermatological physical examination, the computation of the Psoriasis Area and Severity Index (PASI, used to measure the severity and extent of psoriasis) and the Disease Activity Score computed on 44 joints (DAS-44) correlated with the erythrocyte sedimentation rate (ESR) during the first hour (DAS44-ESR). Active rheumatic disease was defined as a DAS44-ESR >3.7; moderate activity as a DAS44 of $\leq 3.7$ to $>2.4$; low activity as a DAS44 of $\leq 2.4$ to $\geq 1.6$; and remission was defined as a DAS44-ESR of $<1.6$. Laboratory values measured included C-reactive protein (CRP, normal range $0-0.5 \mathrm{mg} / \mathrm{dL}$ ) and erythrocyte sedimentation rate (ESR).

Quality of life (QoL) was evaluated using the Dermatology Life Quality Index (DLQI), calculated as the sum of the score for each question, with a maximum score of 30 and a minimum of 0 , and with a higher score denoting greater impairment of QoL. ${ }^{22,23}$ The patients were also asked to evaluate pain on a visual analogue scale (pain VAS) ranging from 0 (absence of pain) to 100 (the worst pain) $\mathrm{mm}$.

\section{Study outcomes}

The primary study outcomes included the mean change from baseline in PASI score, the proportion of patients who achieved $75 \%, 90 \%$ and $100 \%$ reductions in PASI score (PASI75, PASI90 and PASI100, respectively) and mean change from baseline in modified Nail Psoriasis Severity Index (mNAPSI) score (in patients with nail involvement).
The secondary outcomes were the changes from baseline in DAS-44-ESR score, Tender Joint Count (TJC) and Swollen Joint Count (SJC), DLQI, CRP and pain VAS.

The safety analysis included evaluation of all treatment-emergent adverse events (TEAEs).

\section{Statistical analysis}

Discrete variables were summarized by frequencies and percentages. Continuous variables were summarized using standard measures of central tendency and dispersion, including mean, standard deviation, standard error, median, minimum and maximum. Differences in mean values at different time points were analysed using a paired Student's $t$-test with a statistical significance level of $P=0.05$. A general linear model was used for multivariate analysis. Statistical analyses were performed using SPSS version 21.0 (IBM clinical software, IBM SPSS Statistics for Windows, Version 21.0., IBM SPSS Statistics for Windows, Version 21.0. Armonk, NY: IBM Corp., Armonk, NY, USA).

\section{Results}

\section{Baseline demographic and clinical characteristics}

A total of 153 patients, 78 males and 75 females, with a mean age 55 years were enrolled in the study (Table 1). At baseline, the mean duration of psoriasis was $16.3(\mathrm{SD} \pm 12.3)$ years and the mean duration of psoriatic arthritis was 8.8 years $(\mathrm{SD} \pm 7.8)$. Fifty-six patients $(37 \%)$ had nail psoriasis. Most patients $(85 \%)$ had previously received treatment with methotrexate, and 43 patients were in combination treatment with methotrexate.

\section{Primary outcome}

Certolizumab significantly reduced mean PASI score from baseline (8.73) to weeks 12, 24 and 52 (4.10, 2.35 and 1.22, respectively; Fig. 1). The mean change in PASI score from baseline was -4.45 at week $12,-6.30$ at week 24 and -7.58 at week 52 (all $P<0.001$ ). At week 12, $29 \%$ of patients had achieved PASI75, $19 \%$ had achieved PASI90 and 19\% had achieved PASI100 (Fig. 2). At week 24, PASI75 had been achieved by 57\%, PASI90 by $42 \%$ and PASI 100 by $38 \%$; respective values at week 52 were $80 \%, 63 \%$ and $60 \%$ (Fig. 2). The proportion of patients with a PASI score of $\leq 3$ was $69.6 \%$ at week 24 and $83.3 \%$ at week 52 .

Among patients with nail psoriasis $(n=56)$, there were significant decreases in mNAPSI mean score from a baseline at all time points $(P<0.001)$. The baseline mNAPSI mean $( \pm \mathrm{SD})$ score was $14.64( \pm 20.86)$, with changes from baseline of -5.69 $( \pm 8.54)$ at week $12,-8.77( \pm 14.51)$ at week 24 and -12.92 $( \pm 22.10)$ at week 52 .

\section{Secondary outcomes}

Certolizumab was associated with significant improvements in joint involvement (Table 2). Mean DAS44-ESR scores were 
Table 1 Patient demographic and baseline characteristics

\begin{tabular}{ll}
\hline Parameter & $\begin{array}{l}\text { Full analysis set } \\
(\boldsymbol{N}=\mathbf{1 5 3})\end{array}$ \\
\hline $\begin{array}{l}\text { Age at first certolizumab treatment, } \\
\text { mean years } \pm \text { SD }\end{array}$ & $55.0 \pm 12.4$ \\
Male, $n$ (\%) & $78(50.9)$ \\
\hline Disease duration, mean years \pm SD & \\
Psoriasis & $16.3 \pm 12.3$ \\
Psoriatic arthritis & $8.8 \pm 7.8$ \\
BMI (kg/m ${ }^{2}$ ) mean SD & $28.4(4.67)$ \\
\hline Comorbidity, $\boldsymbol{n}$ (\%) & \\
Hypertension & $68(44.4)$ \\
Hypercholesterolaemia & $54(35.3)$ \\
Hypertriglyceridaemia & $32(20.9)$ \\
Diabetes & $21(13.7)$ \\
Cardiovascular disease & $13(8.5)$ \\
Other & $48(31.4)$ \\
\hline Concomitant treatment, $\boldsymbol{n}(\%)$ & \\
Methotrexate & $43(28.1)$ \\
Other & $1(0.7)$ \\
\hline Previous treatment, $\boldsymbol{n}$ (\%) & \\
Methotrexate & $130(85.0)$ \\
Cyclosporin & $87(56.9)$ \\
Adalimumab & $69(45.1)$ \\
Etanercept & $65(42.5)$ \\
Infliximab & $31(20.3)$ \\
Ustekinumab & $28(18.3)$ \\
Golimumab & $22(14.4)$ \\
Rituximab & $16(10.5)$ \\
Secukinumab & $12(7.8)$ \\
Phototherapy & $29(19.0)$ \\
\hline & \\
\hline & \\
\hline
\end{tabular}

SD, standard deviation; UV, ultraviolet.

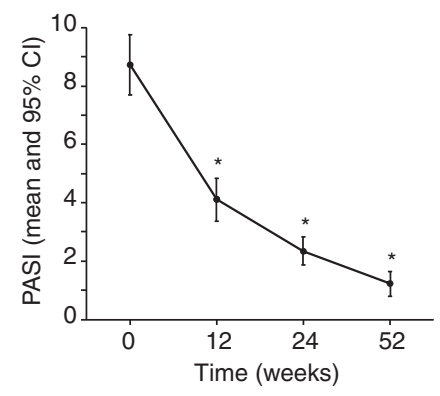

Figure 1 Mean PASI by study visit in the full analysis set $(N=153)$. Cl, confidence interval; PASI, Psoriasis Area and Severity Index. ${ }^{*} P<0.001$ vs. week 0 .

significantly reduced from baseline at weeks 12, 24 and 52 (all $P<0.001$ ). At baseline, $53 \%$ of patients were categorized as having high disease activity (DAS44-ESR > 3.7) and $43 \%$ as having

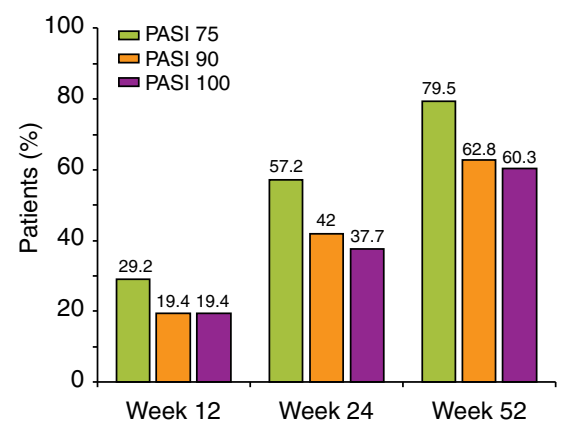

Figure 2 Proportion of patients with a PASI score decrease of $\geq 75 \%$ (PASI 75 ), $\geq 90 \%$ (PASI90) and $100 \%$ (PASI100). Full analysis population ( $n=144$ at week $12, n=138$ at week 24 and $n=78$ at week 52). PASI, Psoriasis Area and Severity Index.

moderate activity (DAS44-ESR $\leq 3.7$ to $>2.4$ ). High and moderate disease activity was found at week 12 in $12 \%$ and $38 \%$ of patients, respectively, at week 24 in $1 \%$ and $20 \%$, and at week 52 in $2 \%$ and $15 \%$ of patients. Mean TJC and SJC also decreased significantly from baseline at weeks 12, 24 and 52 (all $P<0.001$ ). Certolizumab was also associated with significant reductions from baseline in mean pain VAS scores at weeks 12, 24 and 52 (all $P<0.001)$. Mean CRP levels improved from baseline during the study, although the only statistically significant decrease was observed at week $24(P=0.002)$. Patient QoL improved, with significant reduction from baseline in mean DLQI score at each of the three time points $(P<0.001$; Table 2$)$.

On multivariate analysis using a general linear model, the following covariates were found to be significant predictors of the improvement from baseline in PASI score at week 12: psoriasis duration $(P=0.002)$, baseline PASI $(P<0.0001)$, baseline mNAPSI $(P=0.047)$ and baseline pain VAS $(P<0.0001)$.

\section{Safety}

Overall, 21 (14\%) TEAEs were reported during treatment with certolizumab, and six patients withdrew due to adverse events (AEs) (Table 3). AEs leading to discontinuation in one patient each included the following: lack of treatment efficacy in secondary joints, retroperitoneal paraganglioma, worsening of psoriasis, loss of treatment efficacy, breast cancer and surgery. The most commonly reported AEs (in $\geq 2$ patients) were worsening psoriasis $(n=4)$, urinary tract infection $(n=3)$, influenza $(n=2)$ and pneumonia $(n=2)$. No serious AEs were reported.

\section{Discussion}

In this real-world study, certolizumab significantly reduced mean PASI scores from baseline by $5-8$ points over 52 weeks in patients with psoriasis and psoriatic arthritis. Of note, at 52 weeks the proportion of patients with PASI75 was $80 \%$ and PASI90 was $63 \%$. Moreover, $83 \%$ of patients had a PASI score of 
Table 2 Summary of effect of certolizumab on secondary efficacy endpoints

\begin{tabular}{lllll}
\hline Parameter, mean \pm SD & Baseline & Week 12 & Week 24 & Week 52 \\
\hline DAS44-ESR score & $3.88 \pm 0.90$ & $2.60 \pm 0.79$ & $1.96 \pm 0.71$ & $1.78 \pm 0.71$ \\
Change from baseline & - & $-1.28 \pm 0.72$ & $-1.96 \pm 0.88$ & $-2.23 \pm 0.89$ \\
$P$-value & - & $<0.001$ & $<0.001$ & $<0.001$ \\
TJC & $12.50 \pm 9.70$ & $6.56 \pm 6.59$ & $3.53 \pm 4.97$ & $2.86 \pm 4.75$ \\
Change from baseline & - & $-5.94 \pm 5.66$ & $-9.40 \pm 7.47$ & $-9.91 \pm 7.91$ \\
$P$-value & - & $<0.001$ & $<0.001$ & $<0.001$ \\
SJC & $5.57 \pm 4.26$ & $2.25 \pm 2.74$ & $1.05 \pm 2.05$ & $0.94 \pm 1.76$ \\
Change from baseline & - & $-3.32 \pm 3.50$ & $-4.52 \pm 4.19$ & $-4.90 \pm 4.05$ \\
$P$-value & - & $<0.001$ & $<0.001$ & $<0.001$ \\
Pain VAS score & $60.68 \pm 26.51$ & $32.30 \pm 21.17$ & $20.38 \pm 17.78$ & $21.31 \pm 16.14$ \\
$\quad$ Change from baseline & - & $-28.39 \pm 21.75$ & $-43.07 \pm 23.59$ & $-49.37 \pm 19.81$ \\
$P$-value & - & $<0.001$ & $<0.001$ & $<0.001$ \\
CRP, mg/dL & $4.25 \pm 6.17$ & $3.28 \pm 9.43$ & $2.62 \pm 3.66$ & $2.69 \pm 2.29$ \\
$\quad$ Change from baseline & - & $-1.07 \pm 10.64$ & $-1.90 \pm 6.67$ & $-1.47 \pm 6.35$ \\
$P$-value & - & 0.233 & 0.002 & 0.059 \\
DLQI score & $15.75 \pm 5.44$ & $8.65 \pm 4.51$ & $5.76 \pm 3.60$ & $4.80 \pm 3.60$ \\
$\quad$ Change from baseline & - & $-7.02 \pm 5.37$ & $-10.18 \pm 5.85$ & $-10.82 \pm 6.16$ \\
$P$-value & - & $<0.001$ & $<0.001$ & $<0.001$ \\
\hline
\end{tabular}

CRP, C-reactive protein; DAS44-ESR, Disease Activity Score computed on 44 joints correlated with the erythrocyte sedimentation rate during the first hour; DLQI, Dermatological Life Quality Index; SD, standard deviation; SJC, Swollen Joint Count; TJC, Tender Joint Count; VAS, visual analogue scale.

Table 3 Summary of adverse events

\begin{tabular}{ll} 
& Safety set $(\mathbf{N}=\mathbf{1 5 3})$ \\
\hline Treatment-emergent AEs, $n(\%)$ & $21(13.7)$ \\
AEs leading to treatment withdrawal & $6(3.9)$ \\
\hline AEs occurring in $\geq 2$ patients, $n$ (\% of patients experiencing AEs) \\
Worsening psoriasis & $4 / 21(19.0)$ \\
Urinary tract infection & $3 / 21(14.3)$ \\
Influenza & $2 / 21(9.5)$ \\
Pneumonia & $2 / 21(9.5)$ \\
\hline
\end{tabular}

$\mathrm{AE}$, adverse event.

$\leq 3$, indicating a good response to treatment in the majority of patients. Significant improvements were seen for all analysed parameters (except for CRP levels) at all study time points.

Certolizumab was associated with a rapid onset of effect, with a significant decrease from baseline in PASI observed by week 12. This rapid onset of action was confirmed by the secondary outcome analyses, with significant decreases seen in the following scores within 12 weeks of starting treatment: DAS44-ESR, TJC, SJC, pain VAS and DLQI. Indeed, the improvement in quality of life, with a reduction in DLQI score of -7 after only 12 weeks' treatment, was clinically important $\left(\geq \sim 4^{20}\right)$.

The rapid and sustained efficacy of certolizumab in this study is consistent with findings of phase II and III clinical trials of certolizumab in patients with moderate-to-severe plaque psoriasis, in which the key endpoints were PASI75 responder rate and Physician's Global Assessment responder rate. ${ }^{14-16}$ Certolizumab $400 \mathrm{mg}$ gave significant clinical improvements in a 12 -week phase II trial in 176 patients. ${ }^{16}$ In the phase III CIMPASI-1 and CIMPASI-2 trials, certolizumab (400 or $200 \mathrm{mg}$ ) every 2 weeks was significantly more effective than placebo, and results were maintained through to week $48 .{ }^{14}$ In the CIMPACT trial, certolizumab (200 or $400 \mathrm{mg}$ ) every 2 weeks was associated with clinically meaningful differences in PASI75 response rates compared with placebo (61-67\% vs. $5.0 \%$ ), and was superior (at a 400-mg dose) or non-inferior (at a 200-mg dose) to etanercept $50 \mathrm{mg}$ twice weekly (61-67\% vs. $53 \%) .{ }^{15}$ In a pooled analysis of these three phase III trials (CIMPASI-1, CIMPASI-2 and CIMPACT), which included 850 patients who received treatment with certolizumab or placebo, both doses of certolizumab gave statistically significant and clinically meaningful improvements in PASI75 and PGA responder rates compared with placebo after 16 weeks' treatment ( $75-80 \%$ vs. $8 \%$ and $55-64 \%$ vs. $3 \%$, respectively; all $P<0.0001) .^{24}$ The rapid and sustained efficacy of certolizumab in our patient cohort can reassure clinicians that many of their patients are likely to experience improvements with 'real-world' use of the drug at a rheumatologic dose (i.e. $400 \mathrm{mg}$ every 2 weeks for the first 4 weeks and $200 \mathrm{mg}$ every 2 weeks thereafter) and that its efficacy is not limited to the dosage regimens used in the clinical trials mentioned above.

In the current study, in addition to improving PASI score, certolizumab significantly improved joint involvement. At 12, 24 and 52 weeks, there were significant reductions from baseline in the mean DAS44-ESR score, TJC and SJC, as well as in the mean pain VAS and DLQI score. Certolizumab also rapidly improved joints, skin, nail disease, and other signs and symptoms of psoriatic arthritis in the 24-week, phase III, randomized, double-blind 
RAPID-PsA trial in 409 patients. ${ }^{13}$ In that trial, the American College of Rheumatology 20\% (ACR20) response rate was significantly higher with certolizumab than placebo after 1 week of treatment $[\sim 21-23 \%$ vs. $8 \%, P<0.001$ (values estimated from a graph)], and significant between-group differences were observed at every time point through to the end of the trial (24 week ACR20 response rates $52-58 \%$ vs. $24 \%, P<0.001$ ). Certolizumab also significantly improved psoriatic skin involvement and physical function from baseline. The efficacy of certolizumab was maintained over 4 years in the open-label extension of the RAPID-PsA trial. ${ }^{25}$ Evidence from two realworld studies further supports the effectiveness of certolizumab for the joint involvement of psoriatic arthritis and the skin component of those with skin involvement/psoriasis. ${ }^{17,18}$

Approximately one-third of the certolizumab recipients in the certolizumab phase III clinical trials had previously received biologic therapy. ${ }^{14,15}$ It is interesting to note that in this study, a larger proportion of patients [105/153 (69\%)] had previously received biologics. Thus, certolizumab was efficacious even in a pretreated patient population.

Nail involvement is common in psoriasis, particularly in patients with psoriatic arthritis, and can be difficult to treat. ${ }^{26}$ In the current study, certolizumab significantly improved the mean mNAPSI score from a baseline of $14.64-8.95$ by week 12 , and the mean score continued to improve over the duration of the study. These results are consistent with findings in the abovementioned RAPID-PsA trial, in which certolizumab significantly reduced the mean mNAPSI from baseline ( $\sim 3$ points) to week 24 by $\sim 2$ points, ${ }^{13}$ and in which these improvements were maintained to week $96^{27}$ and week $216^{25}$ in the open-label extension. In a small trial in patients with psoriatic arthritis and severe nail damage, certolizumab treatment reduced the mean NAPSI score from 50.3 at baseline to 20.5 at week 24 and 10.0 at week $52 .{ }^{19}$

Of particular interest in the current study, multivariate analysis showed several covariates (psoriasis duration, baseline PASI, baseline DAS44-ESR and baseline pain VAS) were predictive of the improvement in PASI score from baseline to week 12. This indicates that patients with longer duration of disease and higher PASI, DAS44-ESR and pain VAS scores at baseline may experience the greatest benefit from certolizumab treatment.

Real-world studies such as the one reported here offer the opportunity to evaluate the tolerability of treatment in routine clinical practice. Certolizumab was generally well tolerated, with infections being the most common AEs (after exclusion of worsening of disease), although none were considered serious. A previous observational study $(n=44)$ in psoriatic arthritis patients also found no serious infections, ${ }^{18}$ and certolizumab phase II-III clinical trials also reported the majority of AEs to be of mild-tomoderate severity. ${ }^{13-16}$ The profile of the most common AEs in this study was slightly different to that of the latter trials, where nasopharyngitis, upper respiratory tract infections and headache were generally the most common AEs. ${ }^{13-16}$

\section{Limitations}

This study is not without limitations, including its retrospective nature and the relatively small sample size. Nevertheless, our real-world observational study provides reassurance that the clinical effectiveness of certolizumab seen in the randomized controlled trials can be generalized to a more diverse patient population, such as is seen in routine clinical practice.

\section{Conclusion}

In routine clinical practice, certolizumab provided rapid and sustained improvements in psoriasis and joint symptoms and was well tolerated in Italian patients with psoriasis and psoriatic arthritis. Also, we noticed that certolizumab pegol is a good option in naive or multi-failure patients indifferently.

\section{Acknowledgements}

We would like to thank Marie Cheeseman, who wrote the outline of this manuscript on behalf of Springer Healthcare Communications, and Toni Dando of Springer Healthcare Communications who wrote the first draft. This medical writing assistance was funded by Tor Vergata University, Rome.

\section{Author contributions}

$\mathrm{AD}$ was the coordinator of the study, and contributed to study design and critical revision of the manuscript; LB was a principal investigator; MCF enrolled patients and was involved in critical revision of the manuscript; all other authors enrolled patients. All authors have read and approved the final draft.

\section{References}

1 Boehncke W-H, Schön MP. Psoriasis. Lancet 2015; 386: 983-994.

2 Kim WB, Jerome D, Yeung J. Diagnosis and management of psoriasis. Can Fam Physician 2017; 63: 278-285.

3 Santus P, Rizzi M, Radovanovic D et al. Psoriasis and respiratory comorbidities: the added value of fraction of exhaled nitric oxide as a new method to detect, evaluate, and monitor psoriatic systemic involvement and therapeutic efficacy. Biomed Res Int 2018; 23: 3140682.

4 Bocheńska K, Smolińska E, Moskot M, Jakóbkiewicz-Banecka J, GabigCimińska M. Models in the research process of psoriasis. Int J Mol Sci 2017; 18: 2514.

5 Chiricozzi A, Zhang S, Dattola A et al. New insights into the pathogenesis of cutaneous autoimmune disorders. J Biol Regul Homeost Agents 2012; 26: $165-170$.

6 Menter A, Strober BE, Kaplan DH et al. Joint AAD-NPF guidelines of care for the management and treatment of psoriasis with biologics. J Am Acad Dermatol 2019; 80: 1029-1072.

7 Lee EB, Amin M, Bhutani T, Wu JJ. Emerging therapies in psoriasis: a systematic review. Cutis 2018; 101: 5-9.

8 Ronholt K, Iversen L. Old and new biological therapies for psoriasis. Int J Mol Sci 2017; 18: 2297.

9 Gossec L, Smolen JS, Ramiro S et al. European League Against Rheumatism (EULAR) recommendations for the management of psoriatic arthritis with pharmacological therapies: 2015 update. Ann Rheum Dis 2016; 75: 499-510.

10 Campanati A, Benfaremo D, Luchetti MM et al. Certolizumab pegol for the treatment of psoriasis. Expert Opin Biol Ther 2017; 17: 387-394. 
11 Lee JU, Shin W, Son JY, Yoo KY, Heo YS. Molecular basis for the neutralization of tumor necrosis factor alpha by certolizumab pegol in the treatment of inflammatory autoimmune diseases. Int J Mol Sci 2017; 18: E228.

12 Goel N, Stephens S. Certolizumab pegol. MAbs 2010; 2: 137-147.

13 Mease PJ, Fleischmann R, Deodhar AA et al. Effect of certolizumab pegol on signs and symptoms in patients with psoriatic arthritis: 24 -week results of a phase 3 double-blind randomised placebo-controlled study (RAPID-PsA). Ann Rheum Dis 2014; 73: 48-55.

14 Gottlieb AB, Blauvelt A, Thaci D et al. Certolizumab pegol for the treatment of chronic plaque psoriasis: Results through 48 weeks from 2 phase 3 , multicenter, randomized, double-blinded, placebo-controlled studies (CIMPASI-1 and CIMPASI-2). J Am Acad Dermatol 2018; 79: 302-314 e306.

15 Lebwohl M, Blauvelt A, Paul C et al. Certolizumab pegol for the treatment of chronic plaque psoriasis: results through 48 weeks of a phase 3 , multicenter, randomized, double-blind, etanercept- and placebo-controlled study (CIMPACT). J Am Acad Dermatol 2018; 79: 266-276 e265.

16 Reich K, Ortonne JP, Gottlieb AB et al. Successful treatment of moderate to severe plaque psoriasis with the PEGylated Fab' certolizumab pegol: results of a phase II randomized, placebo-controlled trial with a re-treatment extension. Br J Dermatol 2012; 167: 180-190.

17 Carubbi F, Fidanza R, Palmieri M et al. Safety and efficacy of certolizumab pegol in a real-life cohort of patients with psoriasis and psoriatic arthritis. J Dermatolog Treat 2019; 1-6.

18 Dattola A, Cannizzaro MV, Mazzeo M, Bianchi L. Certolizumab pegol in the treatment of psoriasis and psoriatic arthritis: preliminary real-life data. Dermatol Ther (Heidelb) 2017; 7: 485-492.

19 Mazzeo M, Dattola A, Cannizzaro MV, Bianchi L. Nail psoriasis treated with certolizumab pegol in patients with psoriatic arthritis: preliminary observation. Actas Dermosifiliogr 2019; 110: 169-171.

20 Nast A, Gisondi P, Ormerod A et al. European S3-Guidelines on the systemic treatment of psoriasis vulgaris-Update 2015-Short version-EDF in cooperation with EADV and IPC. J Eur Acad Dermatol Venereol 2015; 29: 2277-2294.
21 Gisondi P, Altomare G, Ayala F et al. Italian guidelines on the systemic treatments of moderate-to-severe plaque psoriasis. J Eur Acad Dermatol Venereol 2017; 31: 774-790.

22 Mease PJ. Measures of psoriatic arthritis: tender and swollen Joint Assessment, Psoriasis Area and Severity Index (PASI), Nail Psoriasis Severity Index (NAPSI), Modified Nail Psoriasis Severity Index (mNAPSI), Mander/Newcastle Enthesitis Index (MEI), Leeds Enthesitis Index (LEI), Spondyloarthritis Research Consortium of Canada (SPARCC), Maastricht Ankylosing Spondylitis Enthesis Score (MASES), Leeds Dactylitis Index (LDI), Patient Global for Psoriatic Arthritis, Dermatology Life Quality Index (DLQI), Psoriatic Arthritis Quality of Life (PsAQOL), Functional Assessment of Chronic Illness Therapy-Fatigue (FACIT-F), Psoriatic Arthritis Response Criteria (PsARC), Psoriatic Arthritis Joint Activity Index (PsAJAI), Disease Activity in Psoriatic Arthritis (DAPSA), and Composite Psoriatic Disease Activity Index (CPDAI). Arthritis Care Res (Hoboken) 2011; 63(Suppl 11): S64-S85.

23 Basra MK, Salek MS, Camilleri L, Sturkey R, Finlay AY. Determining the minimal clinically important difference and responsiveness of the Dermatology Life Quality Index (DLQI): further data. Dermatology 2015; 230: 27-33.

24 Blauvelt A, Reich K, Lebwohl M et al. Certolizumab pegol for the treatment of patients with moderate-to-severe chronic plaque psoriasis: pooled analysis of week 16 data from three randomized controlled trials. $J$ Eur Acad Dermatol Venereol 2019; 33: 546-552.

25 van der Heijde D, Deodhar A, FitzGerald O et al. 4-year results from the RAPID-PsA phase 3 randomised placebo-controlled trial of certolizumab pegol in psoriatic arthritis. RMD Open 2018; 4: e000582.

26 Kivelevitch D, Frieder J, Watson I, Paek SY, Menter MA. Pharmacotherapeutic approaches for treating psoriasis in difficult-to-treat areas. Expert Opin Pharmacother 2018; 19: 561-575.

27 Mease P, Deodhar A, Fleischmann R et al. Effect of certolizumab pegol over 96 weeks in patients with psoriatic arthritis with and without prior antitumour necrosis factor exposure. RMD Open 2015; 1: e000119. 